\title{
CytoResc - "CytoSorb" Rescue for critically ill patients undergoing the COVID-19 Cyto- kine Storm: A structured summary of a study protocol for a randomized controlled trial
}

Helena Stockmann ${ }^{1}$, Theresa Keller ${ }^{2}$, Stefan Büttner ${ }^{3}$, Achim Jörres ${ }^{1,4}$, Detlef Kindgen-Milles ${ }^{5}$, Julius Valentin Kunz ${ }^{1}$ Josef Leebmann ${ }^{6}$, Claudia Spies ${ }^{7}$, Karl Träger ${ }^{8}$, Sascha Treskatsch ${ }^{9}$, Alexander Uhrig ${ }^{10}$, Carsten Willam ${ }^{11}$,

Philipp Enghard ${ }^{1 \dagger}$, Torsten Slowinski ${ }^{{ }^{*}+}$ (D) and for the CytoResc Trial Investigators

\begin{abstract}
Objectives: Approximately 8 - $10 \%$ of COVID-19 patients present with a serious clinical course and need for hospitalization, $8 \%$ of hospitalized patients need ICU-treatment. Currently, no causal therapy is available and treatment is purely supportive. The main reason for death in critically ill patients is acute respiratory failure. However, in a number of patients a severe hyperinflammatory response with excessively elevated proinflammatory cytokines causes vasoplegic shock resistant to vasopressor therapy. A new polystyrene-based hemoadsorber (CytoSorb® ${ }^{\oplus}$, Cytosorbents Inc., New Jersey, USA) has been shown to adsorb effectively cytokines and other middle molecular weight toxins this way reducing their blood concentrations. This has been routinely used in clinical practice in the EU for other conditions where a cytokine storm occurs and an observational study has just been completed on COVID-19 patients. We hypothesized that the extracorporeal elimination of cytokines in critically ill COVID-19 patients with suspected hyperinflammation and shock may stabilize hemodynamics and improve outcome. The primary endpoint is time until resolution of vasoplegic shock, which is a well implemented, clinically relevant endpoint in critical care studies.
\end{abstract}

Trial design: Phase IIb, multicenter, prospective, open-label, randomized, 1:1 parallel group pilot study comparing the additional use of "CytoSorb" to standard of care without "CytoSorb".

\footnotetext{
* Correspondence: torsten.slowinski@charite.de
}

${ }^{\dagger}$ Philipp Enghard and Torsten Slowinski contributed equally to this work.

${ }^{1}$ Department of Nephrology and Medical Intensive Care, Charité Universitätsmedizin Berlin, Berlin, Germany

Full list of author information is available at the end of the article

C The Author(s). 2020 Open Access This article is licensed under a Creative Commons Attribution 4.0 International License, which permits use, sharing, adaptation, distribution and reproduction in any medium or format, as long as you give appropriate credit to the original author(s) and the source, provide a link to the Creative Commons licence, and indicate if changes were made. The images or other third party material in this article are included in the article's Creative Commons licence, unless indicated otherwise in a credit line to the material. If material is not included in the article's Creative Commons licence and your intended use is not permitted by statutory regulation or exceeds the permitted use, you will need to obtain permission directly from the copyright holder. To view a copy of this licence, visit http://creativecommons.org/licenses/by/4.0/ The Creative Commons Public Domain Dedication waiver (http://creativecommons.org/publicdomain/zero/1.0/) applies to the data made available in this article, unless otherwise stated in a credit line to the data. 
(Continued from previous page)

Participants: Patients are recruited from the Intensive Care Units (ICUs) of 7 participating centers in Germany (approximately 10 ICUs). All patients aged 18- 80 with positive polymerase chain reaction (PCR) test for SARS-CoV-2, a C-reactive protein $(C R P) \geq 100 \mathrm{mg} / \mathrm{l}$, a Procalcitonin $(P C T)<2 \mathrm{ng} / \mathrm{l}$, and suspected cytokine storm defined via a vasoplegic shock (Norepinephrine $>0.2 \mu \mathrm{g} / \mathrm{min} / \mathrm{kg}$ to achieve a Mean Arterial Pressure $\geq 65 \mathrm{mmHg}$ ). Patients are included irrespective of indication for renal replacement therapy. Suspected or proven bacterial cause for vasoplegic shock is a contraindication.

Intervention and comparator: Within 24 hours after meeting the inclusion criteria patients will be randomized to receive either standard of care or standard of care and additional "CytoSorb" therapy via a shaldon catheter for 3-7 days. Filter exchange is done every 24 hours. If patients receive antibiotics, an additional dose of antibiotics is administered after each change of "CytoSorb" filter in order to prevent underdosing due to "CytoSorb" treatment.

Main outcomes: Primary outcome is time to resolution of vasoplegic shock (defined as no need for vasopressors for at least 8 hours in order to sustain a MAP $\geq 65 \mathrm{mmHg}$ ) in days. Secondary outcomes are 7 day mortality after fulfilling the inclusion criteria, mortality until hospital discharge, Interleukin-6 (IL-6) measurement on day 1 and 3, need for mechanical ventilation, duration of mechanical ventilation, duration of ICU-stay, catecholamine dose on day 1/2/3 after start of "CytoSorb" and acute kidney injury.

Randomization: An electronic randomization will be performed using the study software secuTrial ${ }^{\circledR}$ administered by the Clinical Study Center (CSC) of the Charité - Universitätsmedizin Berlin, Germany. Randomization is done in blocks by 4 stratified by including center.

Blinding (masking): The trial will be non-blinded for the clinicians and patients. The statistician will receive a blinded data set, so that all analyses will be conducted blinded.

Numbers to be randomized (sample size): As this is a pilot study with the goal to examine the feasibility of the study design as well as the intervention effect, no formal sample size calculation was conducted. A total number of approximately 80-100 patients is planned (40-50 patients per group). Safety assessment is done after the inclusion of each 10 patients per randomization group.

Trial Status: Please see the study protocol version from April 24 2020. Recruitment of patients is still pending.

Trial registration: The study was registered on April 272020 in the German Registry of Clinical Trials (DRKS) under the number DRKS00021447.

Full protocol: The full protocol is attached as an additional file, accessible from the Trials website (Additional file 1). In the interest in expediting dissemination of this material, the familiar formatting has been eliminated; this Letter serves as a summary of the key elements of the full protocol.

Keywords: COVID-19, Randomized controlled trial, protocol, cytokine storm, vasoplegic shock, extracorporeal cytokine elimination

\section{Supplementary information}

Supplementary information accompanies this paper at https://doi.org/10. 1186/s13063-020-04501-0.

Additional file 1. Full Study Protocol.

\section{Acknowledgements}

We would like to acknowledge the valuable and straightforward help of Inka Gotthardt, Grit Bugasch, Roman Weimann, Olaf Bender, The-Hoang Do and Alexander Krannich in all parts of trial design, manuscript generation and BMBF application.

\section{Authors' contributions}

TS, PE and HS designed the trial, wrote the study protocol, obtained ethical approval and applied for BMBF funding. TK did the biostatistic design of the trial and wrote the statistical section of the study protocol, ethical approval and BMBF application. HS wrote this manuscript. SB, AJ, DKM, JK, JL, CS, KT, ST and CW substantially contributed to the study conception and revised the work. All authors read and approved the final manuscript.

\section{Funding}

Funding by the German Federal Ministry of Education and Research (BMBF) was applied. A decision is still pending. The BMBF will and had no role in the design of the study, collection, analysis, and interpretation of data and in writing the manuscript.

\section{Availability of data and materials}

All individual patient data on which the results of the publication are based will be made available in anonymized form to scientists who present a reasonable analysis plan. The aim is to make the scientific findings available for other research projects. Data requests should be addressed to: torsten. slowinski@charite.de. For data access, the applicant must sign a data access authorization. Furthermore, the study protocol, the statistical analysis plan, the patient information and the patient consent form will be made available to all interested persons. These documents are available on an external website for 5 years. The data will be made available for a total of 3 months up to a maximum of 5 years.

Ethics approval and consent to participate

The study was approved by the local ethics committee of the Charite Universitätsmedizin Berlin on April 14 2020. (Reference number: Charité EA1/069/20) 
Written informed consent is obtained from all patients. If a patient is not able to give written informed consent himself, and a legal representative exists, the consent of the representative will be obtained in advance. For all other patients incapable of informed consent, a legal guardian will be requested immediately. If the patient regains consciousness during the course of the study, he or she will be informed about the study and can subsequently consent to participate in the study. If the patient does not retrospectively agree with the study, his data will be discarded. If the patient does not regain consciousness, the appointed caregiver decides

retrospectively on participation in the study. If the patient dies before urgent care could be installed, the personal data of the person concerned will be evaluated in an anonymized manner. In this way, the protection of the personality and the data protection interests of the person concerned are safeguarded. Furthermore, this procedure does not compromise the processing objective of the research project.

\section{Consent for publication}

Not applicable

\section{Competing interests}

$H S, P E, T S, J V K, T K, J L, A U, C S$ and $C W$ declare no competing interests. AJ, SB, ST and DKM have received speaker fees from CytoSorbents. KT has received honoraria for lectures and consultations from CytoSorbents Europe.

\section{Author details}

'Department of Nephrology and Medical Intensive Care, Charité Universitätsmedizin Berlin, Berlin, Germany. ${ }^{2}$ Institute for Biometry and Clinical Epidemiology, Charité - Universitätsmedizin Berlin, Berlin, Germany. ${ }^{3}$ Department of Nephrology, Clinic Aschaffenburg-Alzenau, Aschaffenburg, Germany. ${ }^{4}$ Clinic for Nephrology, Transplantation Medicine and Intensive Care Medicine, University Witten/Herdecke Medical Centre,

Cologne-Merheim, Germany. ${ }^{5}$ Department of Anesthesiology, University Hospital Düsseldorf, Heinrich-Heine-University Duesseldorf, Düsseldorf, Germany. ${ }^{6}$ Interdisciplinary Apheresis Center at Passau General Hospital, Passau, Germany. ${ }^{7}$ Department of Anesthesiology and Intensive Care Medicine, Charité - Universitätsmedizin Berlin (CCM, CVK), Berlin, Germany. ${ }^{8}$ Department of Cardiac Anesthesiology, University Hospital UIm, UIm, Germany. ${ }^{9}$ Department of Anesthesiology and Intensive Care Medicine, Charité - Universitätsmedizin Berlin (CBF), Berlin, Germany. ${ }^{10}$ Department of Internal Medicine/Infectious Diseases and Pulmonary Medicine, Charité Universitätsmedizin Berlin, Berlin, Germany. ${ }^{11}$ Department of Internal Medicine 4-Nephrology and Hypertension, Friedrich-Alexander-University Erlangen-Nürnberg (FAU) and University Hospital Erlangen, Erlangen, Germany.

Received: 10 June 2020 Accepted: 11 June 2020

Published online: 26 June 2020

\section{Publisher's Note}

Springer Nature remains neutral with regard to jurisdictional claims in published maps and institutional affiliations. 